ESAIM: COCV 25 (2019) 84

https://doi.org/10.1051/cocv/2018010
ESAIM: Control, Optimisation and Calculus of Variations

www.esaim-cocv.org

\title{
INCORPORATING KNOWLEDGE ON THE MEASUREMENT NOISE IN ELECTRICAL IMPEDANCE TOMOGRAPHY*
}

\author{
Marc Dambrine ${ }^{1, * *}$, Helmut Harbrecht ${ }^{2}$ and Benedicte Puig ${ }^{1}$
}

\begin{abstract}
The present article is concerned with the identification of an obstacle or void of different conductivity which is included in a two-dimensional domain by measurements of voltage and currents at the boundary. In general, the voltage distribution is prescribed and hence deterministic. Whereas, the current distribution is measured and contains measurement errors. We assume that some information is given on these measurement errors which can be described by means of a random field. We exploit this extra knowledge by minimizing a linear combination of the expectation and the variance of the Kohn-Vogelius functional. It is shown how these ideas can be realized in numerical computations. By numerical results, the applicability and feasibility of our approach is demonstrated.
\end{abstract}

Received October 10, 2017. Accepted January 23, 2018.

\section{INTRODUCTION}

Electrical impedance tomography is used in medical imaging to reconstruct the electric conductivity of a part of the body from measurements of currents and voltages at the surface [26]. The same technique is also used in geophysical explorations. An important special case consists in reconstructing the shape of an unknown inclusion or void assuming (piecewise) constant conductivities. In this case, only one pair of current/voltage measurements is necessary, in principle.

The problem under consideration is a special case of the general conductivity reconstruction problem and is severely ill-posed. It has been intensively investigated as an inverse problem. We refer for example to $[1,4,9,21]$ for numerical algorithms and to $[5,17]$ for particular results concerning uniqueness. Moreover, we refer to $[7,8]$ for methods using the full Dirichlet-to-Neumann map at the outer boundary.

In [29], the problem under consideration has been reformulated as a shape optimization problem for the Kohn-Vogelius functional (see [24]). Then, seeking the unknown inclusion is equivalent to seeking the minimizer of an energy functional. Much attention has been spent on the analysis of this approach $([2,3,13])$ and its comparison with a least-squares tracking type functionals. It is also sufficiently versatile to be used in the context of fluid mechanics [6].

Our objective in this article is to take advantage of properties of the noise to construct a deterministic formulation which incorporates this knowledge. We assume that the measured flux is given as a random field that models the measurement errors. We then aim at minimizing a combination of the expectation and the variance

${ }^{*}$ H.H. has been supported by the Swiss National Science Foundation through the project $\mathcal{H}$-matrix based first and second moment analysis.

${ }^{1}$ Université de Pau et des Pays de l'Adour, E2S UPPA, CNRS, LMAP, Pau, France.

2 Departement für Mathematik und Informatik, Universität Basel, Spiegelgasse 1, 4051 Basel, Switzerland.

** Corresponding author: marc.dambrine@univ-pau.fr 


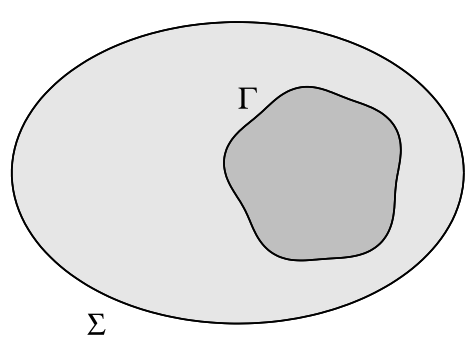

Figure 1 . The domain $D$ and its boundaries $\Gamma$ and $\Sigma$.

of the Kohn-Vogelius functional. As we will show, both quantities can easily be computed via deterministic quantities. The associated shape gradients can likewise be deterministically computed.

The rest of this article is organized as follows. In Section 1, we present the physical model and reformulate the identification problem as shape optimization problem. We introduce the random model and compute the expectation and the variance of the shape functional and their shape gradients. As we will see, both are given by deterministic expressions under some structure assumptions on the random fields under consideration. Then, Section 2 is concerned with the discretization of the shape optimization problem. We assume that the sought inclusion is a starshaped domain which enables us to approximate it by a finite Fourier series. The state equations are reformulated as boundary integral equations which are discretized by means of a fast wavelet boundary element method of linear complexity. In Section 3, we present numerical results which validate the feasibility of the present approach. Finally, in Section 4, we state concluding remarks.

\section{Problem formulation}

\subsection{Physical model}

Let $T \in \mathbb{R}^{d}, d=2,3$, be a simply connected domain with boundary $\Sigma=\partial T$ and assume that an unknown simply connected inclusion $S$ with regular boundary $\Gamma=\partial S$ is located inside the domain $T$ satisfying $\operatorname{dist}(\Sigma, \Gamma)>0, c f$. Figure 1. In order to determine the inclusion $S$, we measure the current distribution $g \in H^{-1 / 2}(\Sigma)$ at the boundary $\Sigma$ for a given voltage distribution $f \in H^{1 / 2}(\Sigma)$. Hence, we are seeking a domain $D:=T \backslash \bar{S}$ and an associated harmonic function $u$, satisfying the system of equations

$$
\begin{aligned}
\Delta u=0 & \text { in } D, \\
u=0 & \text { on } \Gamma, \\
u=f, \frac{\partial u}{\partial \mathbf{n}}=g & \text { on } \Sigma .
\end{aligned}
$$

This system is an overdetermined boundary value problem which admits a solution only for the true inclusion $S$. In accordance with e.g. [25], the inclusion's boundary $\Gamma$ is uniquely determined from $f \neq 0$ and $g$. Nonetheless, it is well known that the problem of finding the inclusion's boundary $\Gamma$ is severely ill-posed. Especially, the measured data $g$ contain noise.

\subsection{Formulation as shape optimization problem}

Following [29], we introduce the auxiliary harmonic functions $v$ and $w$, satisfying

$$
\begin{array}{rlrlrl}
\Delta v & =0 & \Delta w & =0 & & \text { in } D, \\
v & =0 & w & =0 & & \text { on } \Gamma, \\
\frac{\partial v}{\partial \mathbf{n}} & =g & w & =f & & \text { on } \Sigma,
\end{array}
$$


and consider the following shape optimization problem

$$
J(D)=\int_{D}\|\nabla(v-w)\|^{2} \mathrm{~d} \mathbf{x}=\int_{\Sigma}\left(g-\frac{\partial w}{\partial \mathbf{n}}\right)(v-f) \mathrm{d} \sigma \rightarrow \inf .
$$

Herein, the infimum has to be taken over all domains which have a void with sufficiently regular boundary. We refer to [29] for the existence of optimal solutions with respect to this shape optimization problem.

The shape gradient to (1.2) has also been computed in [29]. For variation fields $\mathbf{V}: \mathbb{R}^{d} \rightarrow \mathbb{R}$, being sufficiently smooth, it holds that

$$
\delta J(D)[\mathbf{V}]=\int_{\Gamma}\langle\mathbf{V}, \mathbf{n}\rangle\left[\left(\frac{\partial v}{\partial \mathbf{n}}\right)^{2}-\left(\frac{\partial w}{\partial \mathbf{n}}\right)^{2}\right] \mathrm{d} \sigma .
$$

see also [29]. Given an inclusion $\Sigma$ such the overdetermined boundary problem (1.1) has a solution, the necessary first order optimality condition $\delta J(D)[\mathbf{V}]=0$ is satisfied for all admissible variations $\mathbf{V}$. Notice that the shape Hessian for (1.2) has been computed and analyzed in [13].

\subsection{Random model}

We shall now assume that we have some knowledge on the errors which are caused by the measurement of $g$. Then, we can model $g$ as a random field. To that end, let $(\Omega, \mathcal{S}, \mathbb{P})$ be a complete probability space and assume that $g: \Sigma \times \Omega \rightarrow \mathbb{R}$ is a random field which belongs to the Bochner space $L_{\mathbb{P}}^{2}\left(\Omega, H^{-1 / 2}(\Sigma)\right)$.

Let us recall for the reader's convenience the definition of Bochner spaces. Consider a real number $p \geq 1$. Then, for a Banach space $X$, the Bochner space $L_{\mathbb{P}}^{p}(\Omega, X)$ consists of all functions $v: \Omega \rightarrow X$ whose norm

$$
\|v\|_{L_{\mathbb{P}}^{p}(\Omega, X)}:= \begin{cases}\left(\int_{\Omega}\|v(\cdot, \omega)\|_{X}^{p} \mathrm{~d} \mathbb{P}(\omega)\right)^{1 / p}, & p<\infty, \\ \underset{\omega \in \Omega}{\operatorname{ess} \sup _{\omega}\|v(\cdot, \omega)\|_{X},} & p=\infty,\end{cases}
$$

is finite. If $p=2$ and $X$ is a Hilbert space, then the Bochner space is isomorphic to the tensor product space $L_{\mathbb{P}}^{2}(\Omega) \otimes X$.

Since the data $L_{\mathbb{P}}^{2}\left(\Omega, H^{-1 / 2}(\Sigma)\right)$ are random, also the state $v$ will be a random field. It satisfies $v \in L_{\mathbb{P}}^{2}\left(\Omega, H^{1}(\Omega)\right)$ by linearity of the underlying partial differential equation. As a consequence, the shape functional $J$ becomes also a random process.

Two strategies are a priori available to deal with such a random shape functional. The first one consists in minimizing each realization of the objective and then taking an average of the minimizers. This strategy has been presented in the context of Bernoulli's free boundary problem in [11]. Nonetheless, this approach is unrealistic here due to its high computational cost.

We therefore propose in this article to address the second approach. Namely, we minimize an averaged shape functional as considered in [10]. In particular, we will minimize a combination of the expectation and the variance of the shape functional (1.2). In other words, we seek the domain $D$ with inclusion $S$ in $\operatorname{argmin} F$ where the objective is

$$
F(D)=(1-\alpha) \mathbb{E}[J(D, \omega)]+\alpha \mathbb{V}[J(D, \omega)]
$$

or even

$$
F(D)=(1-\alpha) \mathbb{E}[J(D, \omega)]+\alpha \sqrt{\mathbb{V}[J(D, \omega)]},
$$


where the random shape functional reads as

$$
J(D, \omega)=\int_{D}\|\nabla(v(\omega)-w)\|^{2} \mathrm{~d} \mathbf{x}
$$

and the states read as

$$
\begin{array}{rlrlrl}
\Delta v(\omega) & =0 & \Delta w & =0 & & \text { in } D, \\
v(\omega) & =0 & w & =0 & & \text { on } \Gamma, \\
\frac{\partial v}{\partial \mathbf{n}}(\omega) & =g(\omega) & w & =f & & \text { on } \Sigma .
\end{array}
$$

Let us explain the meaning of the averaged objective defined in (1.4) and (1.5), respectively. When the weight $\alpha$ is equal to 0 , we consider only the average value of the Kohn-Vogelius functional. Its minimization means to be good on a regular base, but does not prohibit a flat distribution and, hence, being often with high values of the of the objective. In order to obtain a shape around which the distribution of the objective is more narrow, we can penalize the variance of the Kohn-Vogelius functional by increasing the weight $\alpha$. Notice that the standard deviation scales like the expectation which makes (1.5) better suited to achieve this goal in comparison with (1.4). The range of admissible $\alpha$ is $[0,1)$, since the expectation is neglected when $\alpha=1$. Then, only the reduction of variance matters and not the average value at all, losing all interest in the shape identification.

Concerning the existence of an optimal shape for the minimization of the objective defined in (1.4) or (1.5), the situation is the same than for the original problem: minimizing $J$. In the class of open subsets of $T$, we do not have an existence result. Existence holds once the class of admissible domains is restricted to some class of domains for which one obtains compactness and the continuity of the solution of the Dirichlet problem with respect to the shape (compare [22]): the class of domains with a uniform exterior cone property for example.

For what follows, we should make the assumption that the Neumann data $g$ are given by the expansion

$$
g(\mathbf{x}, \omega)=g_{0}(\mathbf{x})+\sum_{i=1}^{M} g_{i}(\mathbf{x}) Y_{i}(\omega)
$$

where the random variable $Y_{i}(\omega)$ are independent and identically distributed random variables, $Y_{i} \sim Y$, being centered, $\mathbb{E}[Y]=0$, normalized, $\mathbb{V}[Y]=1$, and having finite fourth order moments. Thus, there especially hold the identities

$$
\mathbb{E}[g(\omega)]=g_{0} \quad \text { and } \quad \mathbb{V}[g(\omega)]=\sum_{i=1}^{M} g_{i}^{2}
$$

Note that we have in mind several independent measurements of the current distribution for the same voltage distribution, from which we can derive the sample mean and the sample covariance. Then, assuming that $g(\mathbf{x}, \omega)$ is a Gaussian random field, the expansion (1.8) can be derived from by means of the Karhunen-Loève expansion, see $[18,27]$ for example.

Given the expansion (1.8), the linearity of the state equation (1.7) implies that

$$
v(\mathbf{x}, \omega)=v_{0}(\mathbf{x})+\sum_{i=1}^{M} v_{i}(\mathbf{x}) Y_{i}(\omega),
$$


where $v_{i}$ solves the equation

$$
\Delta v_{i}=0 \text { in } D, \quad v_{i}=0 \text { on } \Gamma, \quad \frac{\partial v_{i}}{\partial \mathbf{n}}=g_{i} \text { on } \Sigma .
$$

In particular, if $g$ is a Gaussian random field, then also $v$ is a Gaussian random field.

\subsection{Expectation and variance of the random shape functional}

We shall show that the expectation and the variance of the random shape functional (1.6) can be computed from deterministic quantities.

Proposition 1.1. It holds

$$
\mathbb{E}[J(D, \omega)]=\sum_{i=1}^{M} \int_{\Sigma} v_{i} g_{i} \mathrm{~d} \sigma+\int_{\Sigma}\left(g_{0}-\frac{\partial w}{\partial \mathbf{n}}\right)\left(v_{0}-f\right) \mathrm{d} \sigma .
$$

Proof. Following the ideas from [10], using Fubini's theorem for non negative functions, the expectation of the random shape functional can be rewritten as

$$
\begin{aligned}
\mathbb{E}[J(D, \omega)] & =\int_{\Omega} \int_{D}\|\nabla(v(\omega)-w)\|^{2} \mathrm{~d} \mathbf{x} \mathrm{d} \mathbb{P}(\omega) \\
& =\int_{D} \int_{\Omega}\|\nabla(v(\omega)-w)\|^{2} \mathrm{~d} \mathbb{P}(\omega) \mathrm{d} \mathbf{x} \\
& =\left.\int_{D}\left[\int_{\Omega}\langle\nabla(v(\mathbf{x}, \omega)-w(\mathbf{x})), \nabla(v(\mathbf{y}, \omega)-w(\mathbf{y}))\rangle \mathrm{d} \mathbb{P}(\omega)\right]\right|_{\mathbf{x}=\mathbf{y}} \mathrm{d} \mathbf{x} .
\end{aligned}
$$

In view of the expansion (1.9), we thus conclude

$$
\begin{aligned}
& \mathbb{E}[J(D, \omega)]=\int_{D}\left[\int _ { \Omega } \left\langle\nabla\left(v_{0}(\mathbf{x})+\sum_{i=1}^{M} v_{i}(\mathbf{x}) Y_{i}(\omega)-w(\mathbf{x})\right)\right.\right. \\
&\left.\left.\nabla\left(v_{0}(\mathbf{y})+\sum_{j=1}^{M} v_{j}(\mathbf{y}) Y_{j}(\omega)-w(\mathbf{y})\right)\right\rangle \mathrm{d} \mathbb{P}(\omega)\right]\left.\right|_{\mathbf{x}=\mathbf{y}} \mathrm{d} \mathbf{x} .
\end{aligned}
$$

Making now use of the fact that $Y_{i} \sim Y$ are independent and identically distributed random variables, we arrive at

$$
\begin{gathered}
\mathbb{E}[J(D, \omega)]=\int_{D}\left[\sum_{i, j=1}^{M}\left\langle\nabla v_{i}(\mathbf{x}), \nabla v_{j}(\mathbf{y})\right\rangle \mathbb{E}\left[Y_{i} Y_{j}\right]+2 \sum_{i=1}^{M}\left\langle\nabla v_{i}(\mathbf{x}), \nabla\left(v_{0}(\mathbf{y})-w(\mathbf{y})\right)\right\rangle \mathbb{E}\left[Y_{i}\right]\right. \\
\left.+\left\langle\nabla\left(v_{0}(\mathbf{x})-w(\mathbf{x})\right), \nabla\left(v_{0}(\mathbf{x})-w(\mathbf{y})\right)\right\rangle\right]\left.\right|_{\mathbf{x}=\mathbf{y}} \mathrm{d} \mathbf{x} \\
=\int_{D}\left[\sum_{i=1}^{M}\left\|\nabla v_{i}(\mathbf{x})\right\|^{2} \mathbb{E}\left[Y^{2}\right]+2 \sum_{i=1}^{M}\left\langle\nabla v_{i}(\mathbf{x}), \nabla\left(v_{0}(\mathbf{x})-w(\mathbf{x})\right)\right\rangle \mathbb{E}[Y]\right. \\
\left.+\left\|\nabla\left(v_{0}(\mathbf{x})-w(\mathbf{x})\right)\right\|^{2}\right] \mathrm{d} \mathbf{x} .
\end{gathered}
$$


Finally, we can exploit that $Y$ is centered and normalized to arrive at

$$
\mathbb{E}[J(D, \omega)]=\int_{D}\left[\sum_{i=1}^{M}\left\|\nabla v_{i}(\mathbf{x})\right\|^{2}+\left\|\nabla\left(v_{0}(\mathbf{x})-w(\mathbf{x})\right)\right\|^{2}\right] \mathrm{d} \mathbf{x} .
$$

Integration by parts and observing $\partial v_{i} / \partial \mathbf{n}=g_{i}$ in accordance with (1.10) yields thus (1.11).

In complete analogy, one can derive a deterministic expression for the variance of the random shape functional.

Proposition 1.2. It holds

$$
\begin{aligned}
\mathbb{V}[J(D, \omega)]=\left(\mathbb{E}\left[Y^{4}\right]-3\right) \sum_{i=1}^{M}\left(\int_{\Sigma} v_{i} g_{i} \mathrm{~d} \sigma\right)^{2} \\
+4 \mathbb{E}\left[Y^{3}\right] \sum_{i=1}^{M}\left(\int_{\Sigma} v_{i} g_{i} \mathrm{~d} \sigma\right)\left(\int_{\Sigma} g_{i}\left(v_{0}-f\right) \mathrm{d} \sigma\right) \\
+2 \sum_{i, j=1}^{M}\left(\int_{\Sigma} v_{i} g_{j} \mathrm{~d} \sigma\right)^{2}+4 \sum_{i=1}^{M}\left(\int_{\Sigma} g_{i}\left(v_{0}-f\right) \mathrm{d} \sigma\right)^{2} .
\end{aligned}
$$

Proof. Due to the knowledge of (1.11), the variance can be computed from the uncentered second moment of the shape functional in accordance with

$$
\mathbb{V}[J(D, \omega)]=\mathbb{E}\left[J(D, \omega)^{2}\right]-\mathbb{E}^{2}[J(D, \omega)] .
$$

The starting point to derive the uncentered second moment is

$$
\begin{aligned}
\mathbb{E}[ & {\left[J(D, \omega)^{2}\right]=\int_{\Omega}\left[\int_{D}\|\nabla(v(\omega)-w)\|^{2} \mathrm{~d} \mathbf{x}\right]^{2} \mathrm{~d} \mathbb{P}(\omega) } \\
& =\int_{D} \int_{D} \int_{\Omega}\|\nabla(v(\mathbf{x}, \omega)-w(\mathbf{x}))\|^{2}\|\nabla(v(\mathbf{y}, \omega)-w(\mathbf{y}))\|^{2} \mathrm{~d} \mathbb{P}(\omega) \mathrm{d} \mathbf{y} \mathrm{d} \mathbf{x} .
\end{aligned}
$$

By inserting again the expansion (1.9) of the random field $v$ and straightforward calculation, we obtain

$$
\begin{aligned}
\mathbb{E}\left[J(D, \omega)^{2}\right]=\int_{D} \int_{D}\left[\sum_{i, j, k, \ell=1}^{M}\left\langle\nabla v_{i}(\mathbf{x}), \nabla v_{j}(\mathbf{x})\right\rangle\left\langle\nabla v_{k}(\mathbf{y}), \nabla v_{\ell}(\mathbf{y})\right\rangle \mathbb{E}\left[Y_{i} Y_{j} Y_{k} Y_{\ell}\right]\right. \\
+4 \sum_{i, j, k=1}^{M}\left\langle\nabla v_{i}(\mathbf{x}), \nabla v_{j}(\mathbf{x})\right\rangle\left\langle\nabla v_{k}(\mathbf{y}), \nabla\left(v_{0}(\mathbf{y})-w(\mathbf{y})\right)\right\rangle \mathbb{E}\left[Y_{i} Y_{j} Y_{k}\right] \\
+\sum_{i, j=1}^{M}\left\{2\left\langle\nabla v_{i}(\mathbf{x}), \nabla v_{j}(\mathbf{x})\right\rangle\left\|\nabla\left(v_{0}(\mathbf{y})-w(\mathbf{y})\right)\right\|^{2}\right. \\
\left.\quad+4\left\langle\nabla v_{i}(\mathbf{x}), \nabla\left(v_{0}(\mathbf{x})-w(\mathbf{x})\right)\right\rangle\left\langle\nabla v_{j}(\mathbf{y}), \nabla\left(v_{0}(\mathbf{y})-w(\mathbf{y})\right)\right\rangle\right\} \mathbb{E}\left[Y_{i} Y_{j}\right] \\
+4 \sum_{i=1}^{M}\left\langle\nabla v_{i}(\mathbf{x}), \nabla\left(v_{0}(\mathbf{x})-w(\mathbf{x})\right)\right\rangle\left\|\nabla\left(v_{0}(\mathbf{y})-w(\mathbf{y})\right)\right\|^{2} \mathbb{E}\left[Y_{i}\right] \\
\left.+\left\|\nabla\left(v_{0}(\mathbf{x})-w(\mathbf{x})\right)\right\|^{2}\left\|\nabla\left(v_{0}(\mathbf{y})-w(\mathbf{y})\right)\right\|^{2}\right] \mathrm{d} \mathbf{y} \mathrm{d} \mathbf{x} .
\end{aligned}
$$


Of course, this deterministic expression can further be simplified by using the independence of the random variable $Y_{i} \sim Y$. Namely, in view of $\mathbb{E}[Y]=0$ and $\mathbb{V}[Y]=1$, it holds

$$
\begin{aligned}
& \mathbb{E}\left[J(D, \omega)^{2}\right]=\mathbb{E}\left[Y^{4}\right] \sum_{i=1}^{M}\left(\int_{D}\left\|\nabla v_{i}(\mathbf{x})\right\|^{2} \mathrm{~d} \mathbf{x}\right)^{2} \\
& +\mathbb{E}\left[Y^{2}\right]^{2}\left[2 \sum_{i \neq j}\left(\int_{D}\left\langle\nabla v_{i}(\mathbf{x}), \nabla v_{j}(\mathbf{x})\right\rangle \mathrm{d} \mathbf{x}\right)^{2}+\sum_{i \neq j} \int_{D}\left\|\nabla v_{i}(\mathbf{x})\right\|^{2} \mathrm{~d} \mathbf{x} \int_{D}\left\|\nabla v_{j}(\mathbf{x})\right\|^{2} \mathrm{~d} \mathbf{x}\right] \\
& +4 \mathbb{E}\left[Y^{3}\right] \sum_{i=1}^{M} \int_{D}\left\|\nabla v_{i}(\mathbf{x})\right\|^{2} \mathrm{~d} \mathbf{x} \int_{D}\left\langle\nabla v_{i}(\mathbf{x}), \nabla\left(v_{0}(\mathbf{x})-w(\mathbf{x})\right)\right\rangle \mathrm{d} \mathbf{x} \\
& +\mathbb{E}\left[Y^{2}\right]\left[2 \sum_{i=1}^{M} \int_{D}\left\|\nabla v_{i}(\mathbf{x})\right\|^{2} \mathrm{~d} \mathbf{x} \int_{D}\left\|\nabla\left(v_{0}(\mathbf{x})-w(\mathbf{x})\right)\right\|^{2} \mathrm{~d} \mathbf{x}\right. \\
& \left.+4 \sum_{i=1}^{M}\left(\int_{D}\left\langle\nabla v_{i}(\mathbf{x}), \nabla\left(v_{0}(\mathbf{x})-w(\mathbf{x})\right)\right\rangle \mathrm{d} \mathbf{x}\right)^{2}\right] \\
& \left(\int_{D}\left\|\nabla\left(v_{0}(\mathbf{x})-w(\mathbf{x})\right)\right\|^{2} \mathrm{~d} \mathbf{x}\right)^{2} \\
& =\mathbb{E}^{2}[J(D, \omega)]+\left(\mathbb{E}\left[Y^{4}\right]-1\right) \sum_{i=1}^{M}\left(\int_{D}\left\|\nabla v_{i}(\mathbf{x})\right\|^{2} \mathrm{~d} \mathbf{x}\right)^{2} \\
& +4 \mathbb{E}\left[Y^{3}\right] \sum_{i=1}^{M}\left(\int_{D}\left\|\nabla v_{i}(\mathbf{x})\right\|^{2} \mathrm{~d} \mathbf{x}\right)\left(\int_{D}\left\langle\nabla v_{i}(\mathbf{x}), \nabla\left(v_{0}(\mathbf{x})-w(\mathbf{x})\right)\right\rangle \mathrm{d} \mathbf{x}\right) \\
& +2 \sum_{i \neq j}\left(\int_{D}\left\langle\nabla v_{i}(\mathbf{x}), \nabla v_{j}(\mathbf{x})\right\rangle \mathrm{d} \mathbf{x}\right)^{2} \\
& +4 \sum_{i=1}^{M}\left(\int_{D}\left\langle\nabla v_{i}(\mathbf{x}), \nabla\left(v_{0}(\mathbf{x})-w(\mathbf{x})\right)\right\rangle \mathrm{d} \mathbf{x}\right)^{2} \\
& =\mathbb{E}^{2}[J(D, \omega)]+\left(\mathbb{E}\left[Y^{4}\right]-3\right) \sum_{i=1}^{M}\left(\int_{D}\left\|\nabla v_{i}(\mathbf{x})\right\|^{2} \mathrm{~d} \mathbf{x}\right)^{2} \\
& +4 \mathbb{E}\left[Y^{3}\right] \sum_{i=1}^{M}\left(\int_{D}\left\|\nabla v_{i}(\mathbf{x})\right\|^{2} \mathrm{~d} \mathbf{x}\right)\left(\int_{D}\left\langle\nabla v_{i}(\mathbf{x}), \nabla\left(v_{0}(\mathbf{x})-w(\mathbf{x})\right)\right\rangle \mathrm{d} \mathbf{x}\right) \\
& +2 \sum_{i, j=1}^{M}\left(\int_{D}\left\langle\nabla v_{i}(\mathbf{x}), \nabla v_{j}(\mathbf{x})\right\rangle \mathrm{d} \mathbf{x}\right)^{2} \\
& +4 \sum_{i=1}^{M}\left(\int_{D}\left\langle\nabla v_{i}(\mathbf{x}), \nabla\left(v_{0}(\mathbf{x})-w(\mathbf{x})\right)\right\rangle \mathrm{d} \mathbf{x}\right)^{2} .
\end{aligned}
$$

Finally, integration by parts yields the desired expression (1.12).

We have a further simplification of (1.12) in the most important situation of Gaussian random fields. 
Corollary 1.3. If $g$ is a Gaussian random field, then

$$
\mathbb{V}[J(D, \omega)]=2 \sum_{i, j=1}^{M}\left(\int_{\Sigma} v_{i} g_{j} \mathrm{~d} \sigma\right)^{2}+4 \sum_{i=1}^{M}\left(\int_{\Sigma} g_{i}\left(v_{0}-f\right) \mathrm{d} \sigma\right)^{2} .
$$

Proof. In the case of a Gaussian random field, the random variables obey the normal law, i.e., $Y \sim \mathcal{N}(0,1)$. By injecting that it thus holds $\mathbb{E}\left[Y^{4}\right]=3$ and $\mathbb{E}\left[Y^{3}\right]=0$, we derive the assertion immediately from (1.12).

This is the expression we will exploit in our numerical examples. Especially, for sake of convenience, we will provide the shape gradient only for the specific formula (1.13) in the next section and not for the general case (1.12).

\subsection{Shape gradient of the expectation and of the variance}

We shall compute next the shape gradient of the expectation and of the variance. For a survey on the shape calculus, we refer the reader to $[12,28,30]$ and the references therein.

Obviously, due to linearity, the shape gradient $\delta(\mathbb{E}[J(D, \omega)])[\mathbf{V}]$ of the shape functional $\mathbb{E}[J(D, \omega)]$ into the direction of the perturbation field $\mathbf{V}$ is just given by $\mathbb{E}[\delta J(D, \omega)[\mathbf{V}]]$. Hence, it is computed according to

$$
\begin{aligned}
\delta(\mathbb{E}[J(D, \omega)])[\mathbf{V}] & =\mathbb{E}[\delta J(D, \omega)[\mathbf{V}]] \\
& =\int_{\Omega} \int_{\Gamma}\langle\mathbf{V}, \mathbf{n}\rangle\left[\left(\frac{\partial v}{\partial \mathbf{n}}(\omega)\right)^{2}-\left(\frac{\partial w}{\partial \mathbf{n}}\right)^{2}\right] \mathrm{d} \sigma \mathrm{d} \mathbb{P}(\omega) \\
& =\int_{\Gamma}\langle\mathbf{V}, \mathbf{n}\rangle\left\{\left.\left[\int_{\Omega} \frac{\partial v}{\partial \mathbf{n}}(\mathbf{x}, \omega) \frac{\partial v}{\partial \mathbf{n}}(\mathbf{y}, \omega) \mathrm{d} \mathbb{P}(\omega)\right]\right|_{\mathbf{x}=\mathbf{y}}-\left(\frac{\partial w}{\partial \mathbf{n}}(\mathbf{x})\right)^{2}\right\} \mathrm{d} \sigma .
\end{aligned}
$$

We insert the expansion (1.9) and exploit again that the random variables $Y_{i} \sim Y$ are independent, identically distributed, centered and normalized to arrive at the final expression:

$$
\delta(\mathbb{E}[J(D, \omega)])[\mathbf{V}]=\int_{\Gamma}\langle\mathbf{V}, \mathbf{n}\rangle\left[\sum_{i=0}^{M}\left(\frac{\partial v_{i}}{\partial \mathbf{n}}\right)^{2}-\left(\frac{\partial w}{\partial \mathbf{n}}\right)^{2}\right] \mathrm{d} \sigma .
$$

Of course, we could alternatively have computed the shape derivative of the deterministic shape functional (1.11), yielding the same result.

In case of the variance of the random shape functional (1.6), the situation becomes somewhat more difficult. It can be derived by using directly the derivative of the shape functional's second uncentered moment:

$$
\delta(\mathbb{E}[J(D, \omega)])[\mathbf{V}]=\delta\left(\int_{\Omega} J^{2}(D, \omega) \mathrm{d} \mathbb{P}(\omega)\right)[\mathbf{V}]=2 \int_{\Omega} J(D, \omega) \delta J(D, \omega)[\mathbf{V}] \mathrm{d} \mathbb{P}(\omega) .
$$

However, since we are mainly interested in Gaussian random fields $g(\mathbf{x}, \omega)$, i.e., $Y_{i} \sim \mathcal{N}(0,1)$ in $(1.8)$ and $(1.9)$, respectively, we will provide only the shape derivative of (1.13).

Proposition 1.4. Assume that it holds $Y_{i} \sim \mathcal{N}(0,1)$ in (1.8). Then, we have

$$
\delta(\mathbb{V}[J(D, \omega)])[\mathbf{V}]=4 \sum_{i, j=1}^{M}\left(\int_{\Sigma} v_{i} g_{j} \mathrm{~d} \sigma\right)\left(\int_{\Gamma}\langle\mathbf{V}, \mathbf{n}\rangle \frac{\partial v_{i}}{\partial \mathbf{n}} \frac{\partial v_{j}}{\partial \mathbf{n}} \mathrm{d} \sigma\right)
$$




$$
+8 \sum_{i=1}^{M}\left(\int_{\Sigma} g_{i}\left(v_{0}-f\right) \mathrm{d} \sigma\right)\left(\int_{\Gamma}\langle\mathbf{V}, \mathbf{n}\rangle \frac{\partial v_{i}}{\partial \mathbf{n}} \frac{\partial v_{0}}{\partial \mathbf{n}} \mathrm{d} \sigma\right) .
$$

Proof. Since only the functions $v_{i}$ 's depend on the domain $D$, the shape derivative of (1.13) reads

$$
\begin{aligned}
\delta(\mathbb{V}[J(D, \omega)])[\mathbf{V}]= & 4 \sum_{i, j=1}^{M}\left(\int_{\Sigma} v_{i} g_{j} \mathrm{~d} \sigma\right)\left(\int_{\Sigma} \delta v_{i} g_{j} \mathrm{~d} \sigma\right) \\
& +8 \sum_{i=1}^{M}\left(\int_{\Sigma} g_{i}\left(v_{0}-f\right) \mathrm{d} \sigma\right)\left(\int_{\Sigma} g_{i} \delta v_{0} \mathrm{~d} \sigma\right),
\end{aligned}
$$

where the local shape derivatives $\delta v_{i}=\delta v_{i}[\mathbf{V}]$ satisfy the boundary value problems

$$
\Delta \delta v_{i}=0 \text { in } D, \quad \delta v_{i}=-\langle\mathbf{V}, \mathbf{n}\rangle \frac{\partial v_{i}}{\partial \mathbf{n}} \text { on } \Gamma, \quad \frac{\partial v_{i}}{\partial \mathbf{n}}=0 \text { on } \Sigma .
$$

Using that $\partial v_{i} / \partial \mathbf{n}=g_{i}$, we obtain for all $i, j=0,1, \ldots, M$ by integration by parts

$$
\int_{\Sigma} \delta v_{i} g_{j} \mathrm{~d} \sigma+\int_{\Gamma} \delta v_{i} \frac{\partial v_{j}}{\partial \mathbf{n}} \mathrm{d} \sigma=\int_{\Sigma} \underbrace{\frac{\partial \delta v_{i}}{\partial \mathbf{n}}}_{=0} v_{j} \mathrm{~d} \sigma+\int_{\Gamma} \frac{\partial \delta v_{i}}{\partial \mathbf{n}} \underbrace{v_{j}}_{=0} \mathrm{~d} \sigma=0 .
$$

This, in view of (1.15), means that

$$
\int_{\Sigma} \delta v_{i} g_{j} \mathrm{~d} \sigma=\int_{\Gamma}\langle\mathbf{V}, \mathbf{n}\rangle \frac{\partial v_{i}}{\partial \mathbf{n}} \frac{\partial v_{j}}{\partial \mathbf{n}} \mathrm{d} \sigma
$$

for all $i, j=0,1, \ldots, M$. By inserting the latter identities into (1.14), we arrive at the desired assertion.

We mention that the shape derivative of the standard deviation is given by the chain rule in accordance with

$$
\delta \sqrt{\mathbb{V}[J(D, \omega)]}[\mathbf{V}]=\frac{\delta(\mathbb{V}[J(D, \omega)])[\mathbf{V}]}{2 \sqrt{\mathbb{V}[J(D, \omega)]}}
$$

\section{Numerical REALizATiON}

Since the numerical realization is based on the adaptation of the classical Kohn-Vogelius approach for electrical impedance tomography, we briefly recall the ingredients and refer to $[13,14]$ for more details.

\subsection{Boundary integral equations}

We will compute the unknown boundary data of the state functions $v$ and $w$ by boundary integral equations. We introduce the single layer and the double layer operator with respect the boundaries $\Phi, \Psi \in\{\Gamma, \Sigma\}$ by

$$
\begin{array}{ll}
\left(V_{\Phi \Psi} u\right)(\mathbf{x}):=-\frac{1}{2 \pi} \int_{\Phi} \log \|\mathbf{x}-\mathbf{y}\| u(\mathbf{y}) \mathrm{d} \sigma_{\mathbf{y}}, & \mathbf{x} \in \Psi \\
\left(K_{\Phi \Psi} u\right)(\mathbf{x}):=\frac{1}{2 \pi} \int_{\Phi} \frac{\left\langle\mathbf{x}-\mathbf{y}, \mathbf{n}_{\mathbf{y}}\right\rangle}{\|\mathbf{x}-\mathbf{y}\|^{2}} u(\mathbf{y}) \mathrm{d} \sigma_{\mathbf{y}}, & \mathbf{x} \in \Psi
\end{array}
$$


For sake of simplicity, we suppose that $\operatorname{diam} D<1$ to ensure that $V_{\Phi \Phi}$ is invertible, $c f$. [23]. Then, the normal derivative of $w$ is given by the Dirichlet-to-Neumann map

$$
\left[\begin{array}{ll}
V_{\Gamma \Gamma} & V_{\Sigma \Gamma} \\
V_{\Gamma \Sigma} & V_{\Sigma \Sigma}
\end{array}\right]\left[\begin{array}{l}
\left.\frac{\partial w}{\partial \mathbf{n}}\right|_{\Gamma} \\
\left.\frac{\partial w}{\partial \mathbf{n}}\right|_{\Sigma}
\end{array}\right]=\left[\begin{array}{cc}
\frac{1}{2}+K_{\Gamma \Gamma} & K_{\Sigma \Gamma} \\
K_{\Gamma \Sigma} & \frac{1}{2}+K_{\Sigma \Sigma}
\end{array}\right]\left[\begin{array}{l}
0 \\
f
\end{array}\right]
$$

$c f$. (1.7). Likewise, the unknown boundary data of $v_{i}$ are determined by

$$
\left[\begin{array}{cc}
V_{\Gamma \Gamma} & -K_{\Sigma \Gamma} \\
-V_{\Gamma \Sigma} & \frac{1}{2}+K_{\Sigma \Sigma}
\end{array}\right]\left[\begin{array}{c}
\left.\frac{\partial v}{\partial \mathbf{n}}\right|_{\Gamma} \\
\left.v\right|_{\Sigma}
\end{array}\right]=\left[\begin{array}{cc}
\frac{1}{2}+K_{\Gamma \Gamma} & -V_{\Sigma \Gamma} \\
-K_{\Gamma \Sigma} & V_{\Sigma \Sigma}
\end{array}\right]\left[\begin{array}{c}
0 \\
g_{i}
\end{array}\right]
$$

\subsection{Boundary element method}

The shape functional and its gradient can be computed from the knowledge of the boundary data of the state equations (1.7) and (1.10). These data are given by the boundary integral equations (2.1) and (2.2). Hence, it is rather convenient to employ a boundary element method to compute the required boundary data of the

state equations. We use a Galerkin discretization by $N_{\Phi}$ piecewise linear functions $\left\{\theta_{i}^{\Phi}\right\}_{i=1}^{N_{\Phi}}$ on each boundary $\Phi \in\{\Sigma, \Gamma\}$. For $\Phi, \Psi \in\{\Sigma, \Gamma\}$, we introduce the system matrices

$$
\begin{aligned}
& \mathbf{V}_{\Phi \Psi}=-\frac{1}{2 \pi}\left[\int_{\Psi} \int_{\Phi} \log \|\mathbf{x}-\mathbf{y}\| \theta_{i}^{\Phi}(\mathbf{y}) \theta_{j}^{\Psi}(\mathbf{x}) \mathrm{d} \sigma_{\mathbf{y}} \mathrm{d} \sigma_{\mathbf{x}}\right]_{i=1, \ldots, N_{\Phi}, j=1, \ldots, N_{\Psi}}, \\
& \mathbf{K}_{\Phi \Psi}=\frac{1}{2 \pi}\left[\int_{\Psi} \int_{\Phi} \frac{\left\langle\mathbf{x}-\mathbf{y}, \mathbf{n}_{\mathbf{y}}\right\rangle}{\|\mathbf{x}-\mathbf{y}\|^{2}} \theta_{i}^{\Phi}(\mathbf{y}) \theta_{j}^{\Psi}(\mathbf{x}) \mathrm{d} \sigma_{\mathbf{y}} \mathrm{d} \sigma_{\mathbf{x}}\right]_{i=1, \ldots, N_{\Phi}, j=1, \ldots, N_{\Psi}},
\end{aligned}
$$

and the mass matrices

$$
\mathbf{M}_{\Phi}=\left[\int_{\Phi} \theta_{i}^{\Phi}(\mathbf{x}) \theta_{j}^{\Phi}(\mathbf{x}) \mathrm{d} \sigma_{\mathbf{x}}\right]_{i, j=1, \ldots, N_{\Phi}},
$$

and the load vectors of Dirichlet data $f_{\Sigma}$ and Neumann data $g_{i, \Sigma}$

$$
\mathbf{f}_{\Sigma}=\left[\int_{\Sigma} \theta_{i}^{\Sigma}(\mathbf{x}) f(\mathbf{x}) \mathrm{d} \sigma_{\mathbf{x}}\right]_{i=1, \ldots, N_{\Sigma}}, \quad \mathbf{g}_{i, \Sigma}=\left[\int_{\Sigma} \theta_{i}^{\Sigma}(\mathbf{x}) g_{i}(\mathbf{x}) \mathrm{d} \sigma_{\mathbf{x}}\right]_{i=1, \ldots, N_{\Sigma}} .
$$

Then, the linear system of equations

$$
\left[\begin{array}{ll}
\mathbf{V}_{\Gamma \Gamma} & \mathbf{V}_{\Sigma \Gamma} \\
\mathbf{V}_{\Gamma \Sigma} & \mathbf{V}_{\Sigma \Sigma}
\end{array}\right]\left[\begin{array}{l}
\mathbf{a}_{\Gamma} \\
\mathbf{a}_{\Sigma}
\end{array}\right]=\left[\begin{array}{cc}
\frac{1}{2} \mathbf{M}_{\Gamma}+\mathbf{K}_{\Gamma \Gamma} & \mathbf{K}_{\Sigma \Gamma} \\
\mathbf{K}_{\Gamma \Sigma} & \frac{1}{2} \mathbf{M}_{\Sigma}+\mathbf{K}_{\Sigma \Sigma}
\end{array}\right]\left[\begin{array}{c}
\mathbf{0} \\
\mathbf{M}_{\Sigma}^{-1} \mathbf{f}_{\Sigma}
\end{array}\right]
$$

gives us the Neumann data $\left.(\partial w / \partial \mathbf{n})\right|_{\Gamma} \approx \sum_{j=1}^{N_{\Gamma}}\left[\mathbf{a}_{\Gamma}\right]_{j} \theta_{j}^{\Gamma}$ on $\Gamma$ and $\left.(\partial w / \partial \mathbf{n})\right|_{\Sigma} \approx \sum_{j=1}^{N_{\Sigma}}\left[\mathbf{a}_{\Sigma}\right]_{j} \theta_{j}^{\Sigma}$ on $\Sigma$ from the Dirichlet data $f$ on $\Sigma$. Likewise, the system

$$
\left[\begin{array}{cc}
\mathbf{V}_{\Gamma \Gamma} & -\mathbf{K}_{\Sigma \Gamma} \\
-\mathbf{V}_{\Gamma \Sigma} & \frac{1}{2} \mathbf{M}_{\Sigma}+\mathbf{K}_{\Sigma \Sigma}
\end{array}\right]\left[\begin{array}{c}
\mathbf{b}_{\Gamma} \\
\mathbf{b}_{\Sigma}
\end{array}\right]=\left[\begin{array}{cc}
\frac{1}{2} \mathbf{M}_{\Gamma}+\mathbf{K}_{\Gamma \Gamma} & -\mathbf{V}_{\Sigma \Gamma} \\
-\mathbf{K}_{\Gamma \Sigma} & \mathbf{V}_{\Sigma \Sigma}
\end{array}\right]\left[\begin{array}{c}
\mathbf{0} \\
\mathbf{M}_{\Sigma}^{-1} \mathbf{g}_{i, \Sigma}
\end{array}\right]
$$

yields the Dirichlet data $\left.v_{i}\right|_{\Gamma}=\sum_{j=1}^{N_{\Gamma}}\left[\mathbf{b}_{\Gamma}\right]_{j} \theta_{j}^{\Gamma}$ on $\Gamma$ and the Neumann data $\left.\left(\partial v_{i} / \partial \mathbf{n}\right)\right|_{\Sigma} \approx \sum_{j=1}^{N_{\Sigma}}\left[\mathbf{b}_{\Sigma}\right]_{j} \theta_{j}^{\Sigma}$ on $\Sigma$ from the given Neumann data $\mathbf{g}_{i, \Sigma}$ on $\Sigma$.

We mention that the appearing system matrices have to be computed only once for each domain while the system (2.4) has to be solved $M+1$ times to get the $v_{i}$ 's from the $g_{i}$ 's. We will use the wavelet Galerkin scheme 
which yields quasi sparse system matrices and, hence, a linear overall complexity with respect to the number $N_{\Gamma}+N_{\Sigma}$ of degrees of freedom. We refer to [20] for all the details on the wavelet based fast solution of boundary integral equations.

\subsection{Approximation of the free boundary}

For the numerical computations, we restrict ourselves to inclusions which are starshaped with respect to the origin $\mathbf{0}$. Then, the inclusion can be parametrized in accordance with

$$
\gamma:[0,2 \pi] \rightarrow \Gamma, \quad \phi \mapsto r(\phi)\left[\begin{array}{c}
\cos \phi \\
\sin \phi
\end{array}\right]
$$

i.e., we can identify the inclusion via a radial function

$$
r(\phi)=a_{0}+\sum_{n=1}^{\infty} a_{n} \cos (n \phi)+a_{-n} \sin (n \phi) \in C_{\mathrm{per}}^{2}([0,2 \pi]),
$$

which depends only on the polar angle. Hence, it is reasonable to make for the sought inclusion the ansatz

$$
r_{N_{r}}(\phi)=a_{0}+\sum_{n=1}^{N_{r}} a_{n} \cos (n \phi)+a_{-n} \sin (n \phi) .
$$

Since $r_{N_{r}}$ admits $2 N_{r}+1$ degrees of freedom $a_{-N_{r}}, a_{1-N_{r}}, \ldots, a_{N_{r}}$, we arrive at a finite dimensional optimization problem in the open set

$$
A_{N_{r}}:=\left\{a_{-N_{r}}, a_{1-N_{r}}, \ldots, a_{N_{r}} \in \mathbb{R}: r_{N_{r}}(\phi)>0, \phi \in[0,2 \pi]\right\} \subset \mathbb{R}^{2 N_{r}+1} .
$$

Hence, via the identification $r_{N_{r}} \Leftrightarrow D_{N_{r}}$, the finite dimensional approximation of shape minimization problem (1.2) reads as find

$$
D_{N_{r}} \in \underset{A_{N_{r}}}{\operatorname{argmin}}(F)
$$

The associated gradient has to be computed with respect to all directions under consideration:

$$
\mathbf{V}(\phi)=\cos \left(N_{r} \phi\right) \mathbf{e}_{r}(\phi), \cos \left(\left(N_{r}-1\right) \phi\right) \mathbf{e}_{r}(\phi), \ldots, \sin \left(\left(N_{r}-1\right) \phi\right) \mathbf{e}_{r}(\phi), \sin \left(N_{r} \phi\right) \mathbf{e}_{r}(\phi) .
$$

Herein, $\mathbf{e}_{r}(\phi)=(\cos \phi, \sin \phi)$ is the radial direction.

We will apply the quasi-Newton method, updated by the inverse BFGS-rule without damping, in combination with a quadratic line-search in order to solve the minimization problem (2.6). For all the details and a survey on available optimization algorithms, we refer to $[15,16,19]$ and the references therein.

\section{Numerical RESUlts}

In our numerical example, we consider $D$ to be the ellipse with semi-axes 0.45 and 0.3 , having a starshaped inclusion in its interior. This inclusion is to be determined from measurements of the Neumann data for the single voltage distribution $f(\mathbf{x})=x_{1}^{2}-x_{2}^{2}$ at the outer boundary $\Sigma$.

We consider the situation that the noisy measurement $g(\mathbf{x}, \omega)$ is a Gaussian random field. Then, the Neumann data $g(\mathbf{x}, \omega)$ are given in accordance with (1.8), being fully described by having normalized Gaussian random 


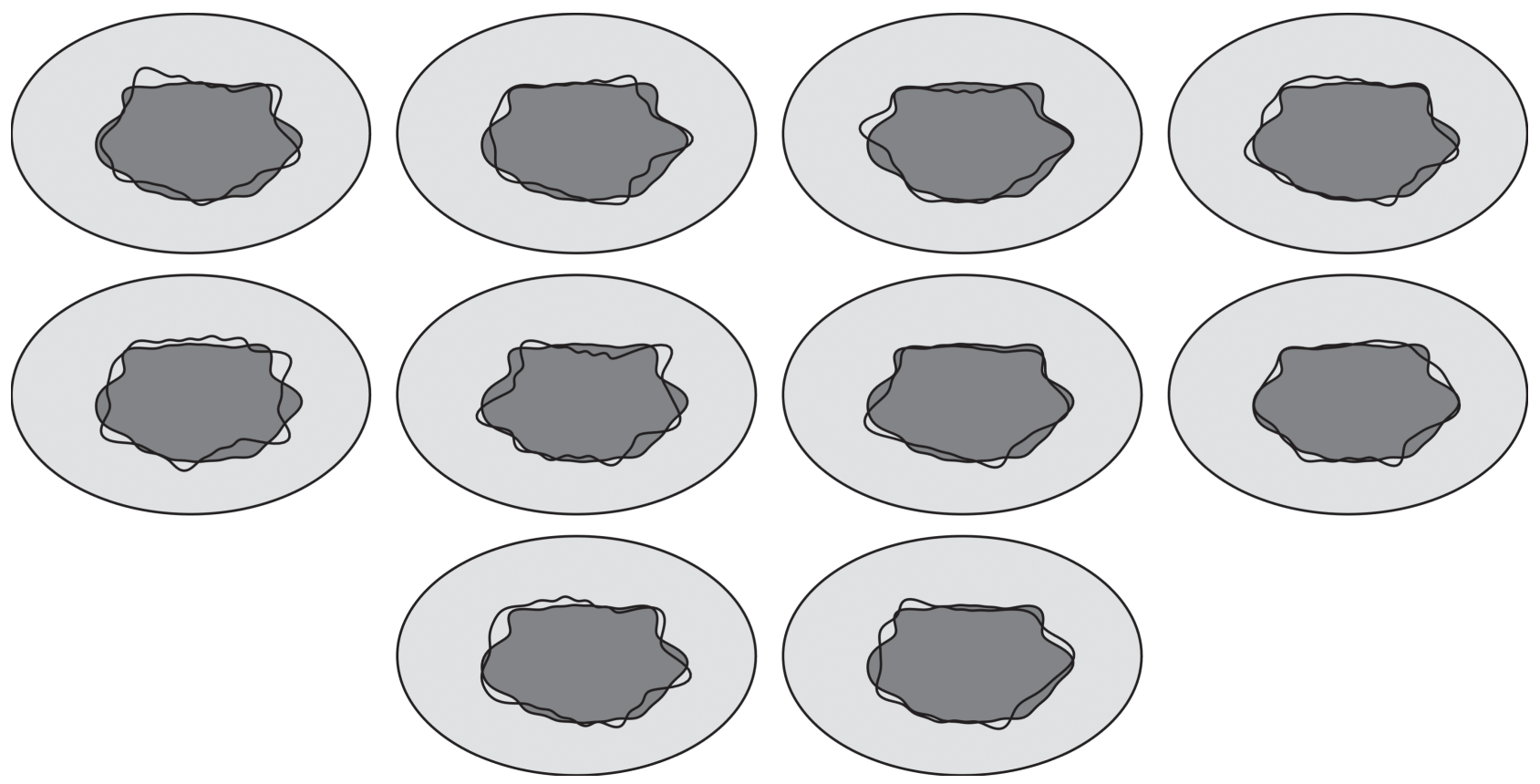

Figure 2. Reconstructions for ten different realizations of the noisy data.

variables $Y_{i} \sim \mathcal{N}(0,1)$ and a certain covariance kernel

$$
\operatorname{Cor}(\mathbf{x}, \mathbf{y})=\int_{\Omega}\left(g(\mathbf{x}, \omega)-g_{0}(\mathbf{x})\right)\left(g(\mathbf{y}, \omega)-g_{0}(\mathbf{y})\right) \mathrm{d} \mathbb{P}(\omega)
$$

We assume for our test that the covariance kernel is a Gaussian kernel

$$
\operatorname{Cor}(\mathbf{x}, \mathbf{y})=\beta \exp \left(-\frac{\|\mathbf{x}-\mathbf{y}\|^{2}}{\ell^{2}}\right)
$$

with correlation length $\ell>0$. Hence, by means of the Karhunen-Loève expansion and an appropriate random number generator, we are able to simulate the Gaussian random field numerically, see [18, 27] for example.

The discretization of the shape optimization problem is as follows. The sought inclusion is approximated by a Fourier expansion of with 33 Fourier coefficients, i.e., it holds $N_{r}=16$ in (2.5). Notice that the sought inclusion cannot be exactly represented by this Fourier expansion. Moreover, the solutions of the boundary integral equations (2.1) and (2.2) are approximately computed by using 512 piecewise linear wavelets per boundary, i.e., it holds $N_{\Sigma}=N_{\Gamma}=512$ in Section 2.2. We use always the circle of radius 0.2 as initial guess and stop the quasi-Newton method after 25 steps since the underlying shape identification problem is severely ill-posed.

\subsection{Classical approach}

The classical approach would be to sample the process $g$ and to minimize the Kohn-Vogelius functional for each realization. In Figure 2, we plotted ten reconstructions which were derived from a single measurement, where the correlation length is set to $\ell=0.1$ and the noise level $\beta$ is chosen such that the perturbation $\left\|g(\omega)-g_{0}\right\|_{L^{2}(\Sigma)}$ is about 5 percent of $\left\|g_{0}\right\|_{L^{2}(\Sigma)}$. We observe a great variance of the reconstructions in Figure 2. In particular, the reconstructions differ mostly considerably from the exact inclusion, which is indicated in dark gray. 


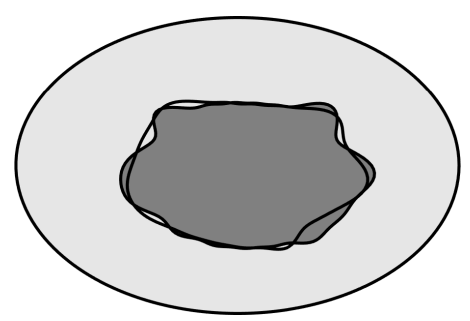

FIGURE 3. Mean of the reconstructed inclusions.

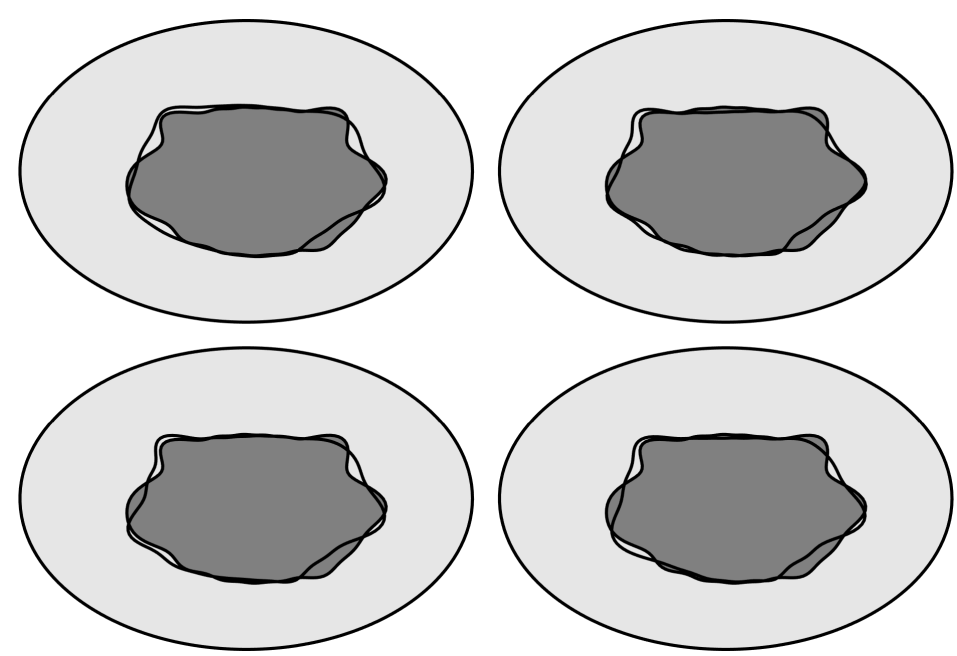

FiguRE 4. Reconstructions for the expected Kohn-Vogelius functional in case of 10 samples.

Since we are using a parametrization (2.5) based on Fourier coefficients, we can compute the mean of the Fourier coefficients of the reconstructions. Doing so for the ten reconstructions found in Figure 2, we obtain the inclusion seen in Figure 3. One clearly observes an improvement of the reconstruction. Nonetheless, this parametrization based notion of the mean shape is not generally possible. In particular, it is computationally extremely expensive.

\subsection{Expected Kohn-Vogelius functional}

To realize the new approach proposed on this article, we repeat the measurement $M$ times, yielding samples $g^{(1)}, g^{(2)}, \ldots, g^{(M)}$ of the unknown random field $g(\omega)$. From these measurements, we compute the sample mean

$$
\bar{g}(\mathbf{x})=\frac{1}{M} \sum_{m=1}^{M} g^{(m)}(\mathbf{x})
$$

to approximate the mean $g_{0}$ in (1.8). The random variation is approximated by means of the Karhunen-Loève expansion with respect to the sample covariance

$$
Q(\mathbf{x}, \mathbf{y})=\frac{1}{M-1} \sum_{m=1}^{M} g^{(m)}(\mathbf{x}) g^{(m)}(\mathbf{y}) .
$$




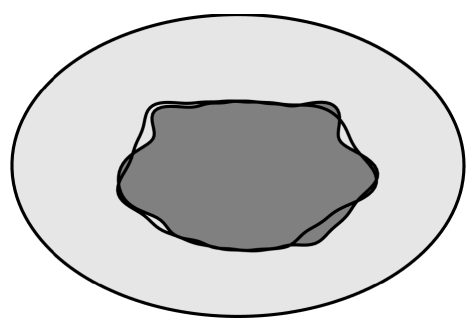

Figure 5. Reconstruction for the expected Kohn-Vogelius functional in case of 100 samples.

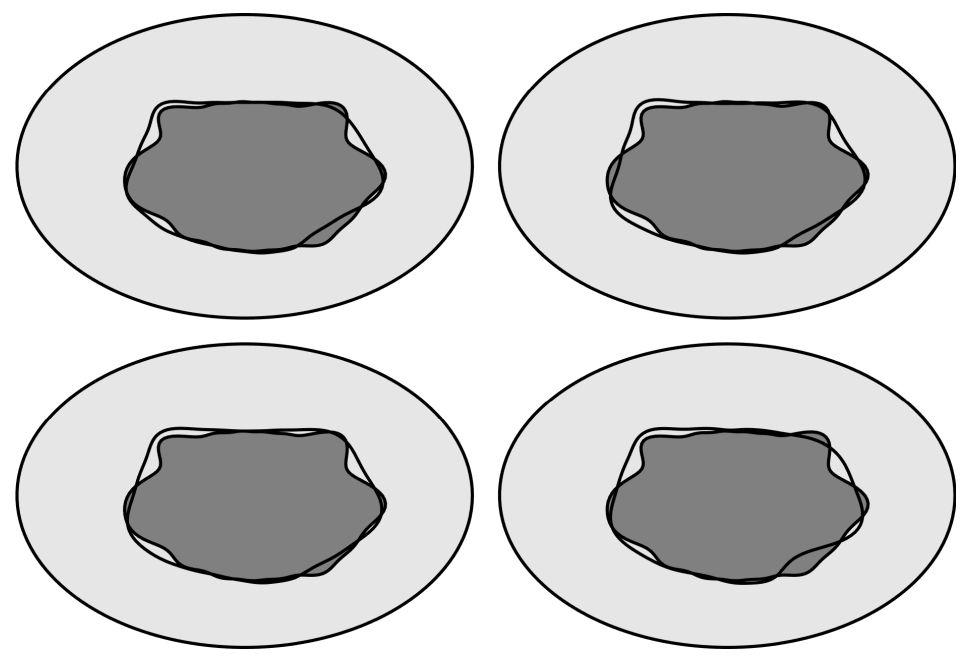

FiguRE 6 . Influence of the coupling parameter: $\alpha=1 / 2,3 / 4,7 / 8,15 / 16$ (from the top to the bottom and from the the left to the right).

If we run the optimization for the expected Kohn-Vogelius functional, i.e., for the objective (1.5) with $\alpha=0$, then we obtain the reconstructions found Figure 4. Here, we have repeated the reconstruction algorithm four times based on $M=10$ samples each. One observes much better reconstructions than those which are obtained from a single measurement. Nonetheless, there is still a slight deviation of the reconstructions. This stems from the fact that ten samples are not sufficient to reliably estimate the expectation and the covariance. The situation changes if we exploit 100 measurements. In this case, we obtain a slightly improved reconstruction, see Figure 5. Especially, there is no more difference when repeating the experiment.

\subsection{Influence of the coupling parameter $\alpha$}

So far, we only considered the minimization of the expected Kohn-Vogelius functional, which means the particular choice $\alpha=0$ in the objective (1.4) and (1.5), respectively. Therefore, we shall study the dependence of the reconstruction algorithm on the coupling parameter $\alpha$. To that end, we choose $M=100$ samples in order to ensure that the reconstruction does not depend on the particular samples.

We consider objective (1.5), since the standard deviation exhibits the same scaling as the expectation. For our test example, both quantities have a similar size for the initial shape. For increasing coupling parameter $\alpha$, the standard deviation of the Kohn-Vogelius functional becomes more and more important compared with its expectation. Nonetheless, the reconstruction is basically the same as seen in Figure 6 . Here, one finds the reconstructions for $\alpha=1 / 2, \alpha=3 / 4, \alpha=7 / 8$, and $\alpha=15 / 16$. 


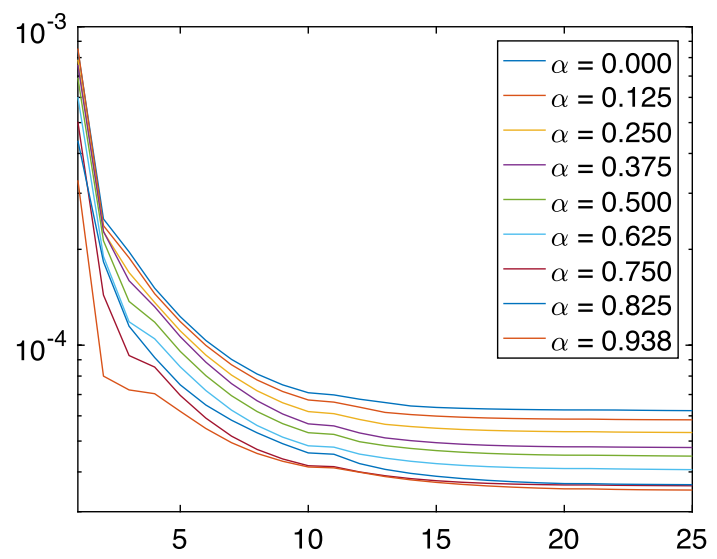

Figure 7. Convergence histories of the objective for different choices of $\alpha$.

What differs for different values of the coupling parameter $\alpha$ is the convergence behaviour of the reconstruction algorithm. Increasing $\alpha$ enforces faster convergence in the beginning of the minimization algorithm, see also Figure 7, where the convergence histories of the objective (1.5) are found for different values of the coupling parameter $\alpha$. Nonetheless, one also figures out that the functional becomes more flat as $\alpha$ increases. Notice that the reconstruction algorithm does not converge anymore for values of $\alpha$ higher than $\alpha=15 / 16$.

\section{Conclusion}

In the present article, we have proposed a method which enables to reconstruct inclusions or void in electrical impedance tomography also in case of very noisy data. Namely, we modeled the measurement data as random field which can approximately be determined from repeated measurements. An objective which combines the expectation and variance of the Kohn-Vogelius functional is then mimimized to reconstruct the sought inclusion. In particular, it is shown that the objective as well as its shape gradient is a deterministic quantity. Numerical results are present which show the capability and feasibility of the proposed approach.

\section{REFERENCES}

[1] L. Afraites, M. Dambrine and D. Kateb, Conformal mapping and inverse conductivity problem with one measurement. ESAIM: COCV. 13 (2007) 163-177.

[2] L. Afraites, M. Dambrine and D. Kateb, Shape methods for the transmission problem with a single measurement. Numer. Funct. Anal. Optim. 28 (2007) 519-551.

[3] L. Afraites, M. Dambrine and D. Kateb, On second order shape optimization methods for electrical impedance tomography. SIAM J. Control Optim. 47 (2008) 1556-1590.

[4] I. Akduman and R. Kress, Electrostatic imaging via conformal mapping. Inverse Probl. 18 (2002) $1659-1672$.

[5] G. Alessandrini, V. Isakov and J. Powell, Local uniqueness in the inverse problem with one measurement. Trans. Am. Math. Soc. 347 (1995) 3031-3041.

[6] M. Badra, F. Caubet and M. Dambrine, Detecting an obstacle immersed in a fluid by shape optimization methods. Math. Models Methods Appl. Sci. 21 (2011) 2069-2101.

[7] M. Brühl, Explicit characterization of inclusions in electrical impedance tomography. SIAM J. Math. Anal. 32 (2001) 13271341.

[8] M. Brühl and M. Hanke, Numerical implementation of two noniterative methods for locating inclusions by impedance tomography. Inverse Probl. 16 (2000) 1029-1042.

[9] R. Chapko and R. Kress, A hybrid method for inverse boundary value problems in potential theory, J. Inv. Ill-Posed Probl. 13 (2005) 27-40.

[10] M. Dambrine, C. Dapogny and H. Harbrecht, Shape optimization for quadratic functionals and states with random right-hand sides. SIAM J. Control Optim. 53 (2015) 3081-3103.

[11] M. Dambrine, H. Harbrecht, M. Peters and B. Puig, On Bernoulli's free boundary problem with a random boundary. Int. J. Uncertain. Quantif. 7 (2017) 335-353.

[12] M. Delfour and J.-P. Zolesio, Shapes and Geometries. SIAM, Philadelphia (2001). 
[13] K. Eppler and H. Harbrecht, A regularized Newton method in electrical impedance tomography using shape Hessian information. Control Cybernet. 34 (2005) 203-225.

[14] K. Eppler and H. Harbrecht, Shape optimization for 3D electrical impedance tomography. In R. Glowinski and J. Zolesio, editors, Free and Moving Boundaries: Analysis, Simulation and Control. Vol. 252 of Lecture Notes in Pure and Applied Mathematics. Chapman \& Hall/CRC, Boca Raton, FL (2007) 165-184.

[15] A.V. Fiacco and G.P. McCormick, Nonlinear Programming: Sequential Unconstrained Minimization Techniques. Wiley, New York (1968).

[16] R. Fletcher, Practical Methods for Optimization. Wiley, New York (1980).

[17] A. Friedman and V. Isakov, On the uniqueness in the inverse conductivity problem with one measurement. Indiana Univ. Math. J. 38 (1989) 563-579.

[18] R. Ghanem and P. Spanos, Stochastic finite elements. A Spectral Approach. Springer, New York (1991).

[19] C. Grossmann and J. Terno, Numerik der Optimierung. B.G. Teubner, Stuttgart (1993).

[20] H. Harbrecht and R. Schneider, Wavelet Galerkin schemes for 2D-BEM. Problems and Methods in Mathematical Physics (Chemnitz, 1999). Edited by J. Elschner, et al. In Vol. 121 of Operator Theory: Advances and Applications. Birkhäuser, Basel (2001) 221-260.

[21] F. Hettlich and W. Rundell, The determination of a discontinuity in a conductivity from a single boundary measurement, Inverse Probl. 14 (1998) 67-82.

[22] A. Henrot and M. Pierre, Shape Variation and Optimization. In Vol. 28 of Tracts in Mathematics. European Mathematical Society (2017).

[23] G. Hsiao and W. Wendland, A finite element method for some equations of first kind. J. Math. Anal. Appl. 58 (1977) $449-481$.

[24] R. Kohn and M. Vogelius, Determining conductivity by boundary measurements. Comm. Pure Appl. Math 37 (1984) $289-298$.

[25] R. Kress, Inverse Dirichlet problem and conformal mapping. Math. Comp. Simul. 66 (2004) 255-265.

[26] W. Lionheart, N. Polydorides and A. Borsic, Electrical impedance tomography: methods, history and applications, edited by D.S. Holder. IOP Series in Medical Physics and Biomedical Engineering. Institute of Physics Publishing (2005) 3-64.

[27] M. Loève, Probability theory. I+II. Fourth edn. In Vol. 45 of Graduate Texts in Mathematics. Springer, New York (1977).

[28] O. Pironneau, Optimal Shape Design for Elliptic Systems. Springer, New York (1984).

[29] J.-R. Roche and J. Sokolowski, Numerical methods for shape identification problems. Control Cybern. 25 (1996) 867-894.

[30] J. Sokolowski and J.-P. Zolesio, Introduction to Shape Optimization. Springer, Berlin (1992). 